\title{
Identification of 4 immune cells and a 5-IncRNA risk signature with prognosis for early-stage lung adenocarcinoma
}

\author{
Lan $\mathrm{Mu}^{1}$, Ke Ding ${ }^{2}$, Ranran Tu${ }^{3}$ and Wei Yang ${ }^{4^{*}}$ (1)
}

\begin{abstract}
Background: Lung cancer is the most common cancer and cause of cancer-related mortality worldwide, increasing evidence indicated that there was a significant correlation between tumors and the long non-coding RNAs (IncRNAs), as well as tumor immune infiltration, but their role in early lung adenocarcinoma (LUAD) are still unclear.

Methods: Gene expression data and corresponding clinical data of early-stage LUAD patients were downloaded from GEO and TCGA databases. 24 kinds of tumor-infiltrating immune cells were analyzed by quantity analysis and univariate cox regression analysis, we divided patients into two subgroups using consensus clustering, recognized the differentially expressed genes (DEGs) in the subgroups, then, established IncRNA risk signature by least absolute shrinkage and selection operator (LASSO) regression.

Results: A total of 718 patients were enrolled in this study, including 246 from GSE31210 dataset, 127 from GSE50081 dataset and 345 from TCGA-LUAD. We identified that Th2 cells, TFH, NK CD56dim cells and Mast cells were prognosisrelated $(p<0.05)$, then established a 5 -InCRNA risk signature (risk score $=0.374600616^{*}$ LINC00857 $+0.173825706^{*}$ LINC01116 + $(-0.021398903)^{*}$ DRAIC $+(-0.113658256)^{*}$ LINC01140 $+(-0.008403702)^{*}$ XIST $)$, and draw a nomogram showed that the signature had a well prediction accuracy and discrimination.
\end{abstract}

Conclusions: We identified 4 immune infiltrating cells related to the prognosis of early-stage LUAD, and established a novel 5 immune-related IncRNA signature for predicting patients' prognosis.

Keywords: Lung adenocarcinoma, Immune infiltration, LncRNA, Prognosis

\section{Background}

Lung cancer is the most common malignant neoplasm, which has also the highest morbidity worldwide in recent years $[1,2]$. Lung cancer includes two main types: nonsmall-cell lung cancer (NSCLC, approximately 85\%) and small-cell lung cancers (SCLC, approximately 15\%) [3], while Lung adenocarcinoma (LUAD) is the most

\footnotetext{
*Correspondence: wyang32@csu.edu.cn

${ }^{4}$ Department of Respiratory Medicine, National Key Clinical Specialty, Xiangya Hospital, Central South University, Changsha 410000, Hunan, China

Full list of author information is available at the end of the article
}

common histological subtypes (about 60\%) of NSCLC [4], which has a five-year survival rate of only $22.1 \%$ [5].

Tumors grow in a complex network consists of epithelial cells, vascular and lymphatic vessels, cytokines and chemokines, as well as infiltrating immune cells, different types of infiltrating immune cells have different effects on tumor progression [6]. Describing the immune infiltration of the tumor microenvironment can untie the role of immune cells and help establishing a well model for predicting tumor prognosis.

Long noncoding RNA (lncRNA) is a class of RNA with a length of more than 200 nucleotides, which cannot code for proteins. Previous studies showed that IncRNA was mainly involved in the regulation of 
gene expression at different levels [7], including splicing, transcription, translation, protein modification regulation, etc. it also plays an important role in tumor immunity, such as antigen releasing and presentation, immune cell migration, immune activation, infiltration in tumor tissues, and some other biological processes [8]. Additionally, more and more lncRNAs have been identified act as an oncogene or tumor suppressor in tumor progression, such as HOTAIR in breast cancer [9], MALAT1 in lung cancer [10], and SNHG15 in papillary thyroid carcinoma [11]. Several studies have been undertaken to identify lncRNA-based signature for predicting overall survival for patients with nonsmall cell lung cancer (NSCLC) [12, 13].

Until now, the treatment strategy and prognosis of lung cancer are mainly based on the TNM staging system. However, LUAD with the same TNM stage may also have different prognosis due to their different types of pathology and diversity of molecule, the role of molecular markers in the diagnosis, prognosis, and therapy of malignancies is well evidenced [14-18]. However, the roles of immune-related lncRNAs in early-stage LUAD are still unclear. Hence, we sought to quantify infiltrating immune cells in tumors and healthy tissues and identify Immune-related lncRNAs signatures. So, we constructed an immune risk signature that could predict the overall survival (OS) for LUAD patients, the immune risk signature can provide additional prognostic information for the TNM staging system, and we hope it will be of great significance to discover new targeted therapies for early-stage LUAD patients.

\section{Materials and methods \\ Early-stage LUAD patient datasets}

Figure 1 showed a flowchart of the steps involved in this study. Gene expression data and corresponding clinical data of LUAD patients were downloaded from the Gene Expression Omnibus (GEO) databases and the cancer genome atlas (TCGA) databases. According to the inclusion criteria of early-stage (stage I and II) LUAD patients. The gene expression data and clinical data of LUAD patients were downloaded from TCGA database by "TCGAbiolinks" package [19] in R (4.0.2). A total of 718 patients were enrolled in our study, including 20 normal samples and 226 patients from GSE31210 dataset, 127 patients from GSE50081 dataset and 345 patients from TCGA-LUAD. Of these, the patients from GSE31210 dataset were used as a training cohort to build a risk signature, while the other two datasets (GSE50081 and TCGA-LUAD) were used as the verifying cohort to validate the signature.

\section{Acquisition and processing of IncRNA expression profile}

We downloaded the data having been processed by the Robust Multichip Average (RMA) algorithm. lncRNA expression data of LUAD patients were obtained by performing a probe reannotation pipeline as previous described [20, 21]. "Homo_sapiens.GRCh38.90.chr.gtf" was used to annotate the probe, and gene biotype was

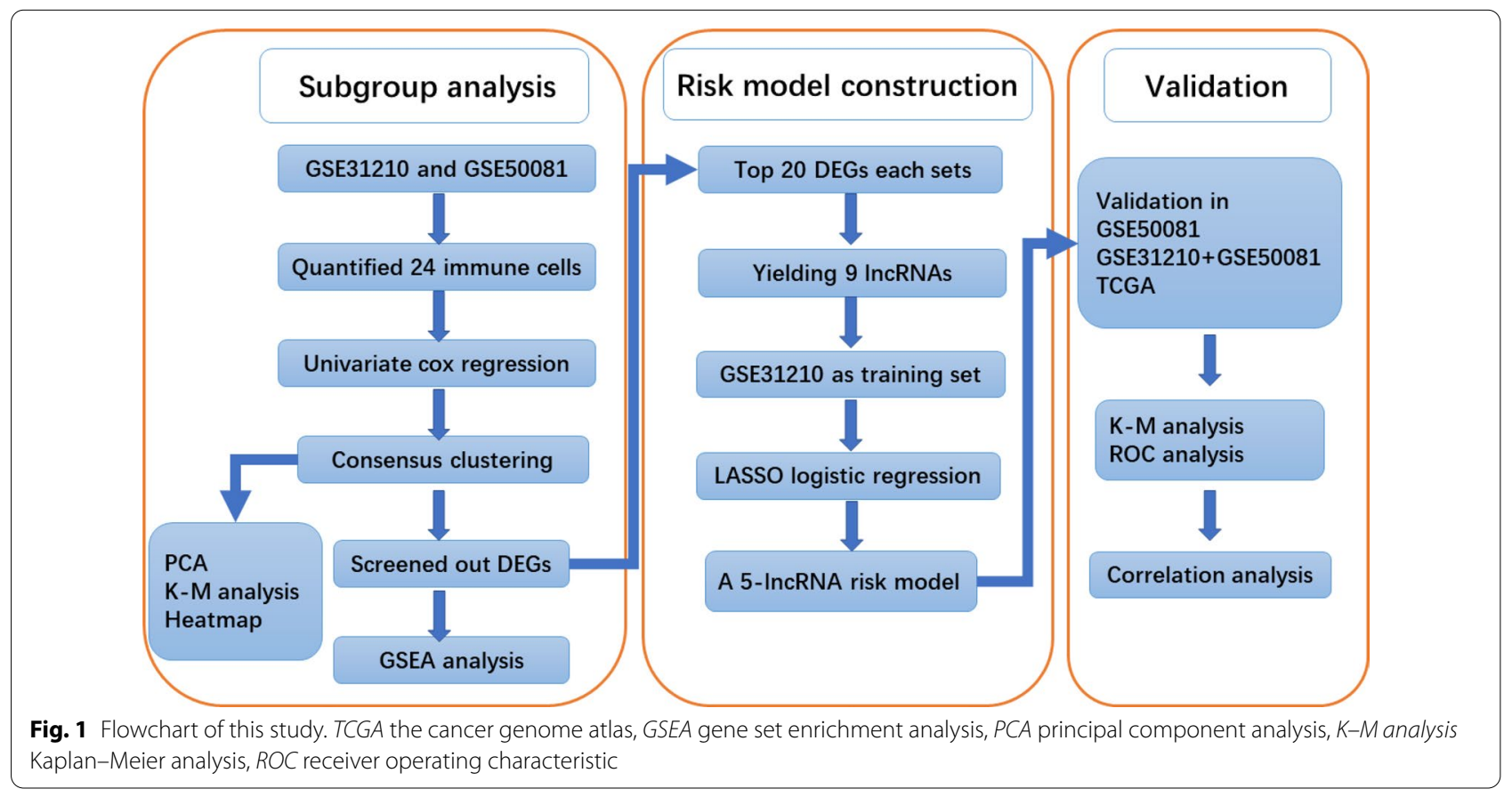


not protein_coding but transcript_biotype was one of the following: "sense_overlapping", "lincRNA", "3prime_overlapping_ncRNA", "processed_transcript", "sense_intronic", "bidirectional_promoter_lncRNA", "non_coding" was considered to be a lncRNA. The average expression value was used when a gene was mapped to multiple probes. As for TCGA data, we downloaded the RNA sequencing expression profiles with the type of counts from the TCGA (https://portal.gdc.cancer.gov), then we extracted lncRNAs using the previous method.

\section{Quantification of infiltrating immune cells}

In terms of quantifying immune infiltrating cells, a review [22] published in Cancer Immunology in 2018 summarized several methods currently used, we used the ssGSEA (single-sample geneset enrichment analysis) as it described to quantify infiltrating immune cells, one of the biggest advantages of this method is that we can customize and quantify the types of immune infiltrating cells by using the "GSVA" package ( $\mathrm{R}$ package). With the widely recognized and used immune cell marker genes provided by Bindea et al. [23], we used these marker genes to extract 24 immune cells' information. Therefore, based on the gene expression data and marker genes, we can use the ssGSEA to quantify infiltrating immune cells.

\section{Differences and correlation in tumor and normal tissues}

To compare the differences of immune cells between LUAD and normal tissue, The "pheatmap" R package and the "vioplot" $R$ package were used for drawing the plots, and we also used the "corrplot" package to show the correlation of immune cells in tumor tissues.

\section{Identification of immune cells and subgroup analysis}

To determine the prognostic value of immune cells, we performed univariate Cox regression analysis on the 24 immune cells in GSE31210 by using the "survival" package in $\mathrm{R}(\mathrm{v} 4.0 .2)$ and measured the hazard ratios (HRs) with 95\% confidence intervals (CIs),it indicated that immune cells were correlated with overall survival (OS) and considered prognostic immune cells with the p-value $<0.05$, immune cells with $H R s<1$ were considered to be risk factors, while HRs $>1$ were protective factors, and further verify with GSE50081. We selected the immune cells that play the same role in the tumor prognosis of the two data sets, and the amount of corresponding immune infiltration cells was used to cluster the LUCD patients into different subgroups with the use the "ConsensusClusterPlus" package. We analyzed the two subgroups using principal component analysis (PCA) with the $\mathrm{R}$ package in $\mathrm{R}$ (v4.0.2) to study the immune cells in different subgroups to judge whether our cluster was correct, and a heatmap was used to show the 24 immune cells in subgroups of the dataset.

\section{Identification of DEGs}

Use the "limma" package to identify the difference between the subgroups of GSE31210 and GSE50081 datasets, that is, to screen out the DEGs (differentially expressed genes) between the immune subgroup with good prognosis and that with poor prognosis. The screening condition were p-value $<0.05$ and $|\operatorname{logFC}| \geq 1$, to pursue their possible functions, we performed GSEA (gene set enrichment analysis) [24] by the "clusterProfiler" $R$ package, and it was considered a statistically significant difference with the $\mathrm{p}$-value $<0.05$.

In addition, in order to determine the prognostic value of lncRNA, lncRNA was extracted from DEGs, and $\operatorname{logFC} \pm$ top 20 were taken from the two data sets respectively, a total of 40 genes $(\mathrm{p}<0.05)$, the intersection was taken, yielding 9 genes.

\section{Identification of hub-genes and risk-score model construction}

Hub-genes were several lncRNAs those closely related to the prognosis of LUAD patients. We proposed GSE31210 as the experimental cohort, and the data of GSE50081 and TCGA were used for validation. Firstly, we combined their gene expression matrices and removed the inter-batch difference using the combat function of "sva"package, resulting in a total of 698 samples, and we've already identified 9 lncRNAs (associated with immunity) through the previous method, next, we did least absolute shrinkage and selection operator (LASSO) logistic regression [25] in 226 samples of GSE31210 to screen out hub-genes and determine their coefficients for developing the risk score model.

We constructed Kaplan-Meier survival curve of LUCD patients and divided the patients into high-risk group and low-risk group according to the cutoff found by the Survminer R package, and the receiver operating characteristic (ROC) and the area under the curve (AUC) were used to assess the prognostic performance of our risk score model.

\section{Building and validation of a nomogram}

A nomogram is a simple graph which used to predict cancer prognosis, we used the previously identified hub genes to construct a nomogram by the "rms" package, then, we draw a calibration plot to verify the nomogram, we calculated the concordance index (C-index) and plotted ROC curves were used to evaluate the predictive ability of the nomogram to measure the nomogram's veracity. 


\section{Results}

Identification of infiltrating immune cells and survival analysis

After the quantification of infiltrating immune cells, GSE31210 was used as the training dataset, we plotted a heatmap and a vioplot to show the amount of 24 immune cells between normal and tumor tissues, the results were shown in Fig 2a, b, 12 kinds of immune cells (B cells, CD8 T cells, Cytotoxic cells, Eosinophils, Mast cells, Neutrophils, NK cells, pDC, T helper cells, Tem, TFH and Tgd) were differentially expressed between them $(\mathrm{p}<0.05)$. And the correlation of immune cells in tumor tissues was shown as Fig. 3, the amount of Th2 cells and Mast cells were negatively correlated with each other in LUAD, while the amount of Th2 cells and NK CD56dim cells were positively correlated.

We performed univariate Cox regression analyses for the expression of 24 immune cells in GSE31210, and further verified with GSE50081. The results were shown in Fig. 4: Th2 cells, TFH, NK CD56dim cells, and Mast cells play the same role in the tumor prognosis of the two datasets, Th2 cells and NK CD56dim cells are protective factors, while TFH and Mast cells are the opposite.

\section{Analysis of subgroup}

According to the amount of the 4 immune cells (Th2 cells, TFH,NK CD56dim cells and Mast cells) identified above, $\mathrm{k}=4$ seemed to be the optimal choice with clustering stability increasing from $\mathrm{k}=2-9$ (Fig. 5b-d) in GSE31210, while $\mathrm{k}=5$ in GSE50081(Fig. 6b-d). However, we found that only when $k=2$, the interference between subgroups was minimal in GSE31210 and GSE50081 (Figs. 5a, 6a). Therefore, the datasets were divided into two subgroups (cluster1\&2), we also analyzed the immune cells in two subgroups by principal component analysis (PCA), it showed a clear distinction between them (Fig. 7a, b), which further indicated our classification was meaningful. In addition, we found that the cluster1 had a significantly shorter overall survival (OS) than those in cluster2 (Fig. 7c, d). And the heatmap (Fig. 8a, b) was plotted to show the amount of 24 immune cells and the distribution of clinicopathologic features between cluster 1 and cluster 2 .

\section{Identification of DEGs and construction of risk score model} We screened out differentially expressed genes (DEGs) (lncRNA) between cluster1 and cluster2 in GSE31210 and GSE50081, then, we performed GSEA (gene set enrichment analysis) to seek out the possible main functional pathways involved, respectively. As a result of 74 enriched pathways involved in GSE31210 and 49 pathways in GSE50081, the top 6 enriched pathways in GSE31210 were Calcium signaling pathway, cAMP signaling pathway, cGMP-PKG signaling pathway, MAPK signaling pathway, Rap1 signaling pathway and Wnt signaling pathway (Fig. 9a), while the top 6 enriched pathways in GSE50081 were Cell cycle, Central carbon metabolism in cancer, Progesterone-madiated oocyte maturation, RNA tansport, Small cell lung cancer and Spliceosome (Fig. 9b), and there were 22 pathways involved in both datasets (Table 1), the above results suggested that the two clusters determined by consensus clustering had different clinical characteristics and prognosis of LUAD. In these two datasets, the main functional pathways seemed to be different, but they also had many of the same pathways (Table 1), it showed that two datasets were more likely to be associated with the malignancy of LUAD. These results indicated that the occurrence, development and prognosis of LUAD involved multiple pathways.

\section{Identification of hub genes and construction of risk score model}

We screened out 9 IncRNAs (associated with immunity) using the method described in methods, they wer e"TXLNGY","LINC00857","LINC00973","LINC01116"," DRAIC","SFTA3","LINC01140","CYP2B7P" and "XIST".
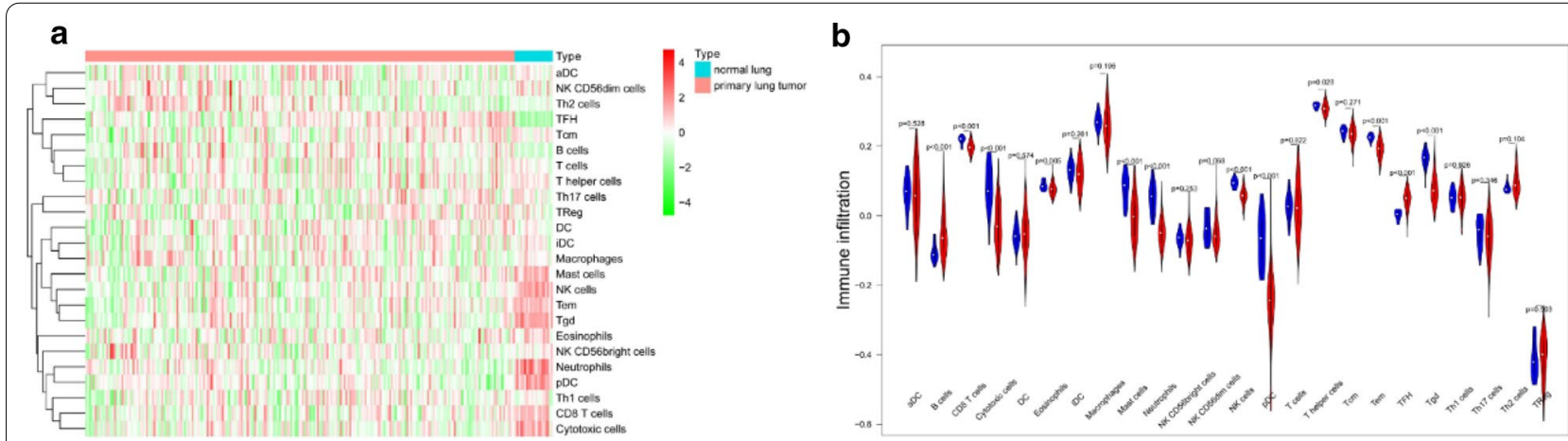

Fig. 2 The amount of 24 immune cells between normal and tumor tissues. a Heatmap; b vioplot 


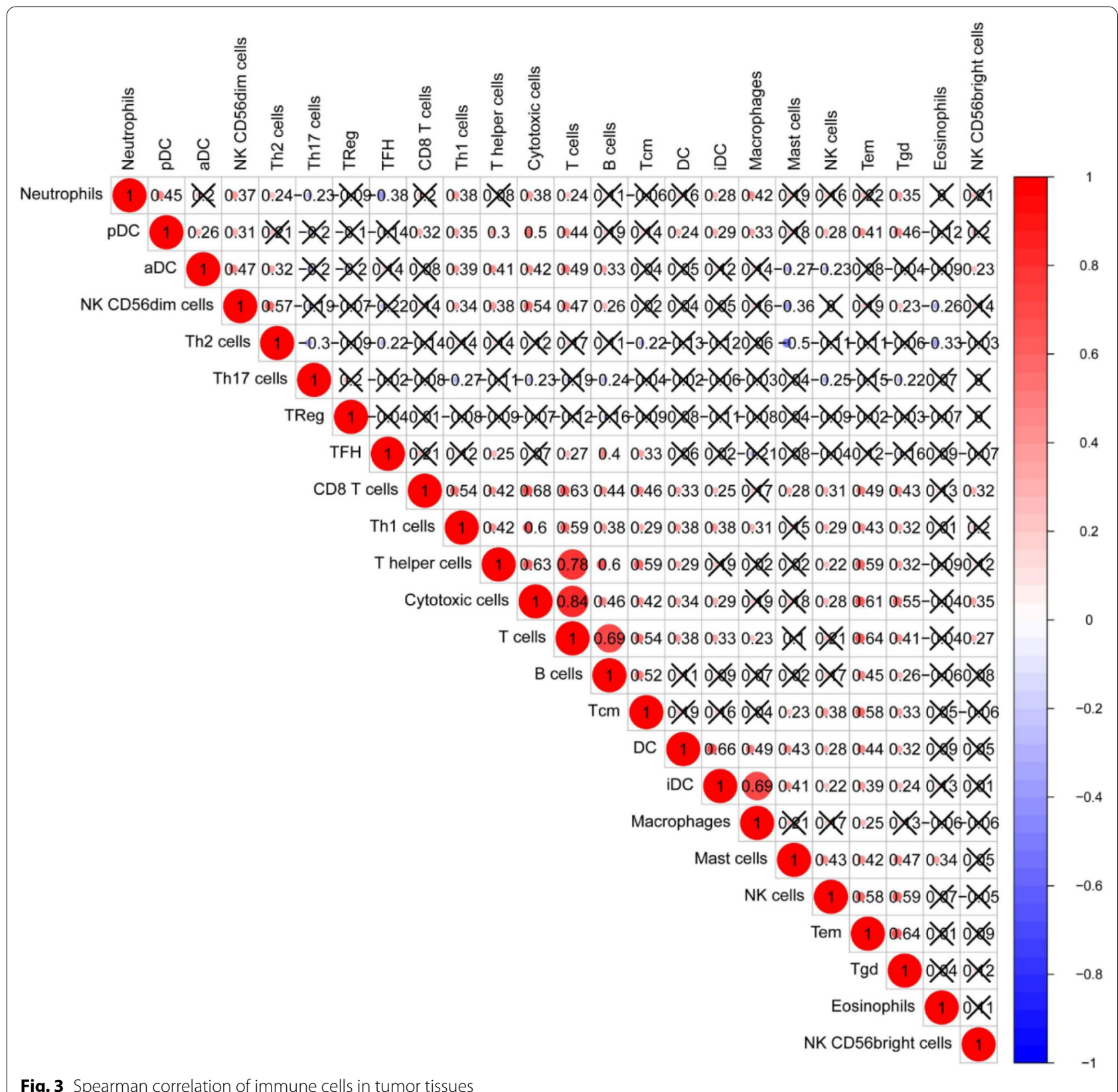

Fig. 3 Spearman correlation of immune cells in tumor tissues

We proposed GSE31210 dataset as the experimental cohort, and the data of GSE50081 and TCGA were used for validation, for further screening out the IncRNA related to prognosis and developing a risk score model, we conducted LASSO logistic regression analysis to the 9 lncRNAs in 226 samples of GSE31210.The LASSO results (Fig. 10a, b) showed that 5 lncRNAs (hub genes) were the powerful prognostic factors, and then constructed the risk score model as: risk score $=0.3746006$ 16 * LINC00857 + 0.173825706 * LINC01116 + $(-0.021$
$398903) *$ DRAIC $+(-0.113658256) *$ LINC01140 $+(-$ $0.008403702) *$ XIST.

We constructed Kaplan-Meier survival curve of early-stage LUCD patients which divided the patients into high-risk and low-risk group according to the optimal cutoff, and the area under the curve (AUC) of the subject's work characteristics (ROC) was used to assess the clinical prognostic ability of this risk score model, therefore, we plotted the risk score distribution, 

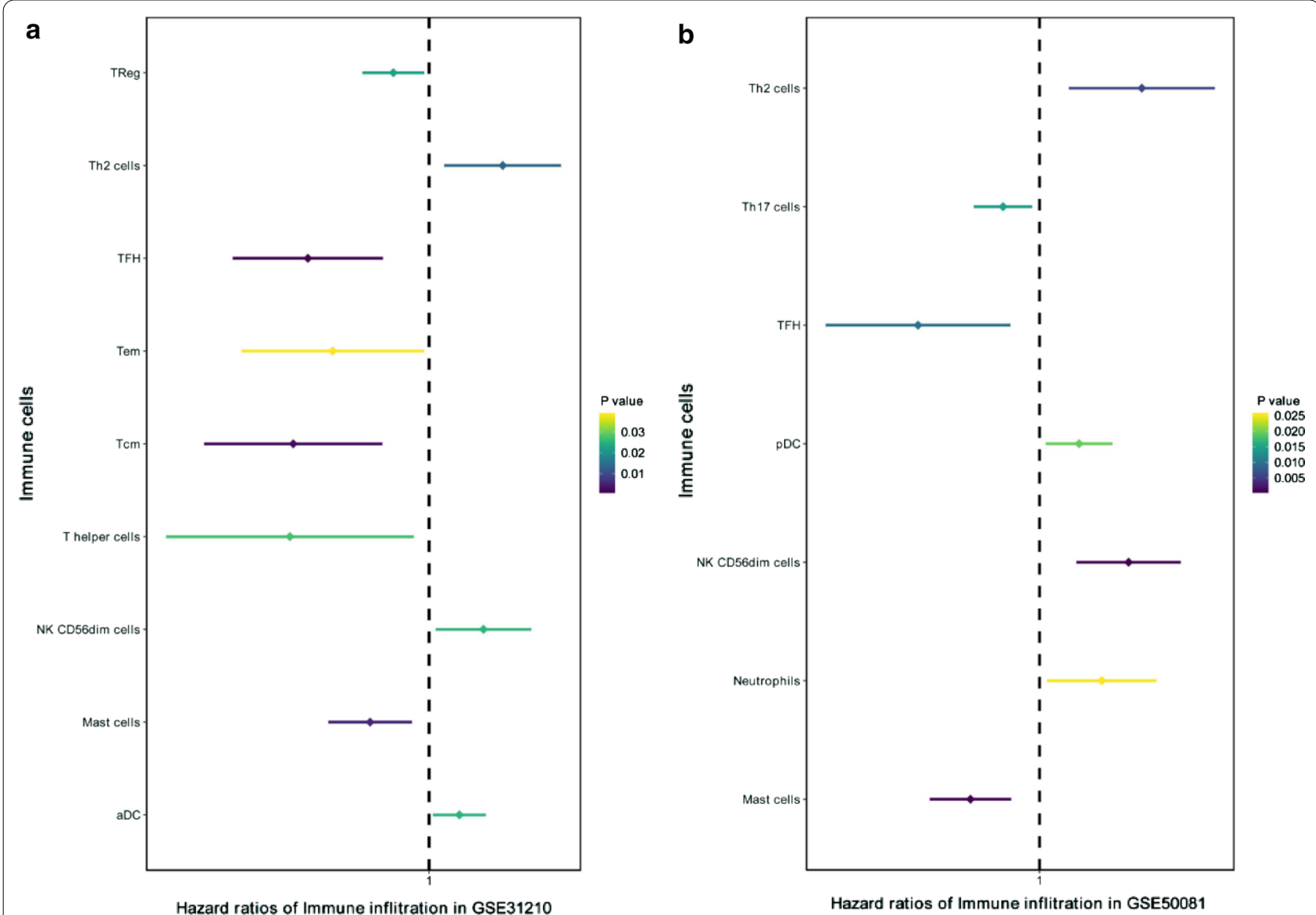

Fig. 4 Forest map of univariate Cox analysis in LUAD. a GSE31210 dataset; b GSE50081 dataset. LUAD lung adenocarcinoma

the time-dependent ROC curve and the survival analysis of the training set (GSE31210), validating set (GSE50081 set, GSE31210 + GSE50081 set and TCGA set) (Fig. 11). The area under the ROC curves (AUCs) of the OS prognostic model were as follows: 12 month AUC: $0.848,36$ month AUC: 0.726, 60 month AUC: 0.709; 12 month AUC: 0. 690, 36 month AUC: 0.755, 60 month AUC: 0.764; 12 month AUC: 0.729, 36 month AUC: $0.731,60$ month AUC: 0.679; 12 month AUC: 0.686, 36 month AUC: 0.625, 60 month AUC: 0.619. In conclusion, it suggested well-prediction performance of the 5-lncRNA signature for survival prediction.

\section{Building a nomogram based on hub genes}

We built a nomogram to predict of 3-year and 5-year OS of LUAD patients (Fig. 12a). Calibration curves and the $\mathrm{C}$-index were used to assess the discrimination and accuracy of the nomogram, the C-index was 0.71121105 in GSE31210 dataset, the C-index was 0.65793781 in GSE50081 dataset, and 0.63457270 in TCGA dataset, the calibration curves for the 3-year and 5-year survivals showed good agreement to the ideal curves in both training set and validation set (Fig. 12b-d), indicating good prediction performance of the nomogram.

\section{Correlation analysis between hub-genes and infiltrating immune cells}

To further verify the correlation between hub-genes and infiltrating immune cells, we performed Spearman correlation analysis using the "ggstatsplot" package, the result showed a significant correlation between them in both GSE31210 dataset and GSE50081 dataset (Fig. 13).

\section{Discussion}

In this study, we identified immune cells and lncRNAs that were strongly correlated with the prognosis of earlystage LUAD patients, thereby deepening the understanding of the immune mechanism of early-stage LUAD. Firstly, we use single-sample Gene Set Enrichment Analysis (ssGSEA) to quantify tumor immune infiltrating cells. Quantitative analysis and univariate COX regression identified 4 immune cells that played the same role in the prognosis of tumors in the two sets. According to the level of immune cells, through consensus clustering, the 


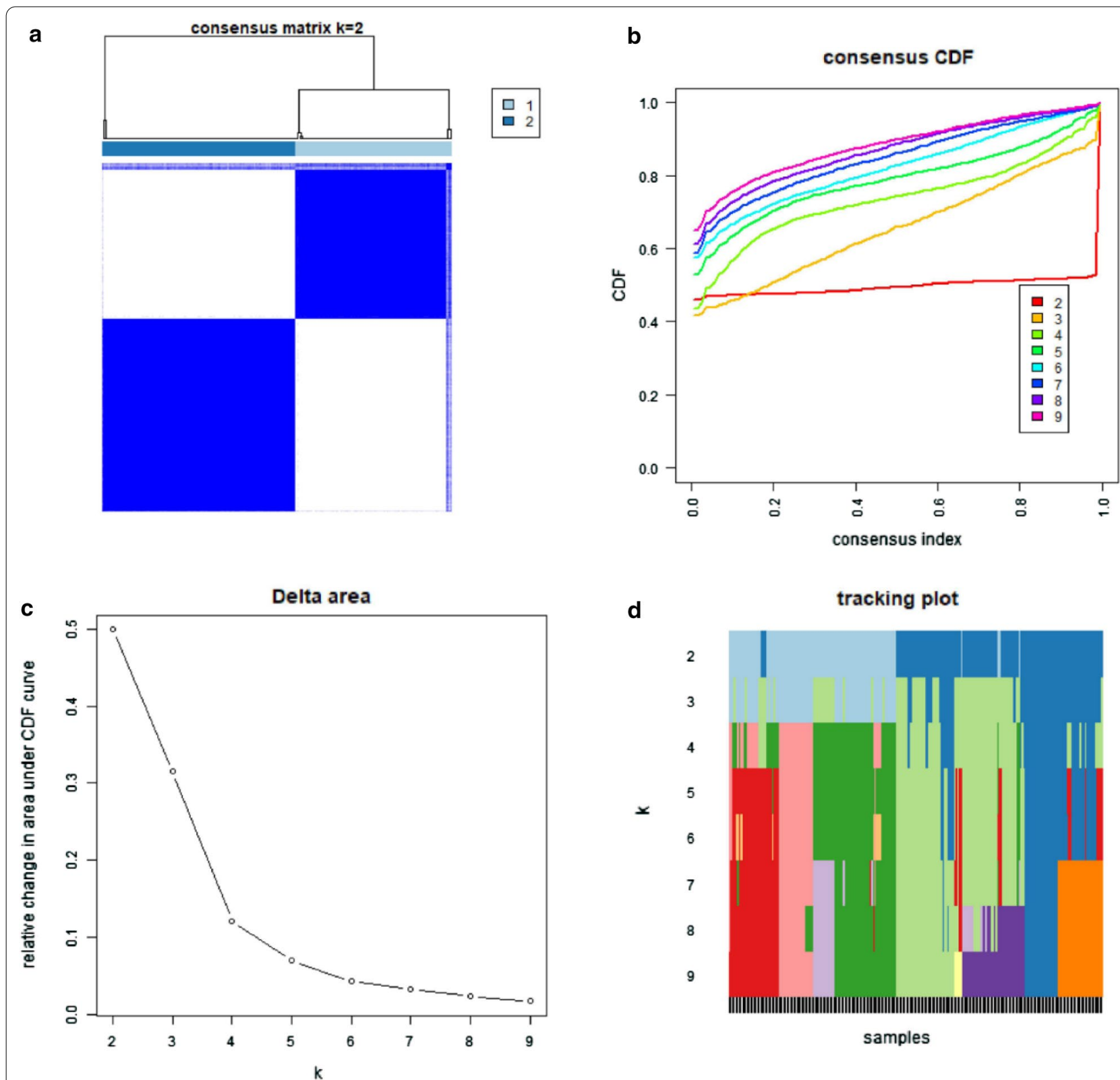

Fig. 5 Identification of consensus clusters by immune cells in GSE31210. a Consensus clustering matrix for $k=2 ; \mathbf{b}$ consensus clustering cumulative distribution function (CDF) for $\mathrm{k}=2-9$; $\mathbf{c}$ relative change in area under CDF curve fork $=2-9$; $\mathbf{d}$ the tracking plot for $\mathrm{k}=2-9$

two sets were divided into two immune subgroups (Cluster1/2) with good or poor prognosis, Cluster1/2 group not only affects the prognosis, but also related to the key signal pathways of LUAD. In addition, according to the differentially expressed genes (DEGs) in the subgroups, on the one hand, GSEA was used to find the enriched pathways that ranked high, and on the other hand, hub genes closely related to prognosis were screened by LASSO regression and a 5 -lncRNAs risk score model was successfully established, and patients were divided into high-risk and low-risk group, the Kaplan-Meier overall survival (OS) curve showed that patients in the low-risk group have a longer OS than those in high-risk group. At the same time, the AUC also showed that the signature had good prognostic value regardless of whether they were in the experimental cohort or the verification cohort, finally, the nomogram also showed a well prediction performance, it further proved that these lncRNAs could potentially serve as biomarkers for the prognosis of early-stage LUAD patients. 


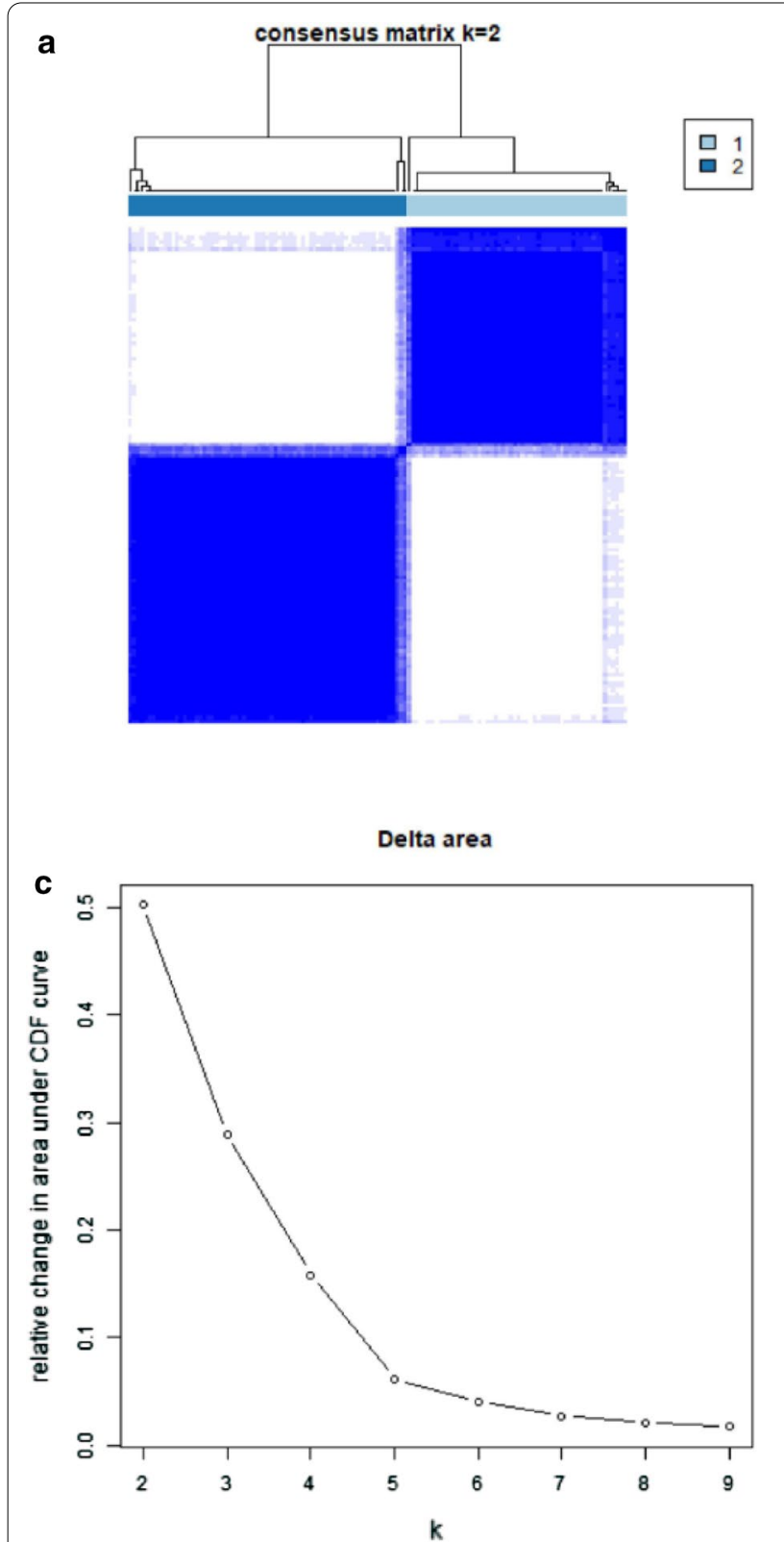

\section{b}
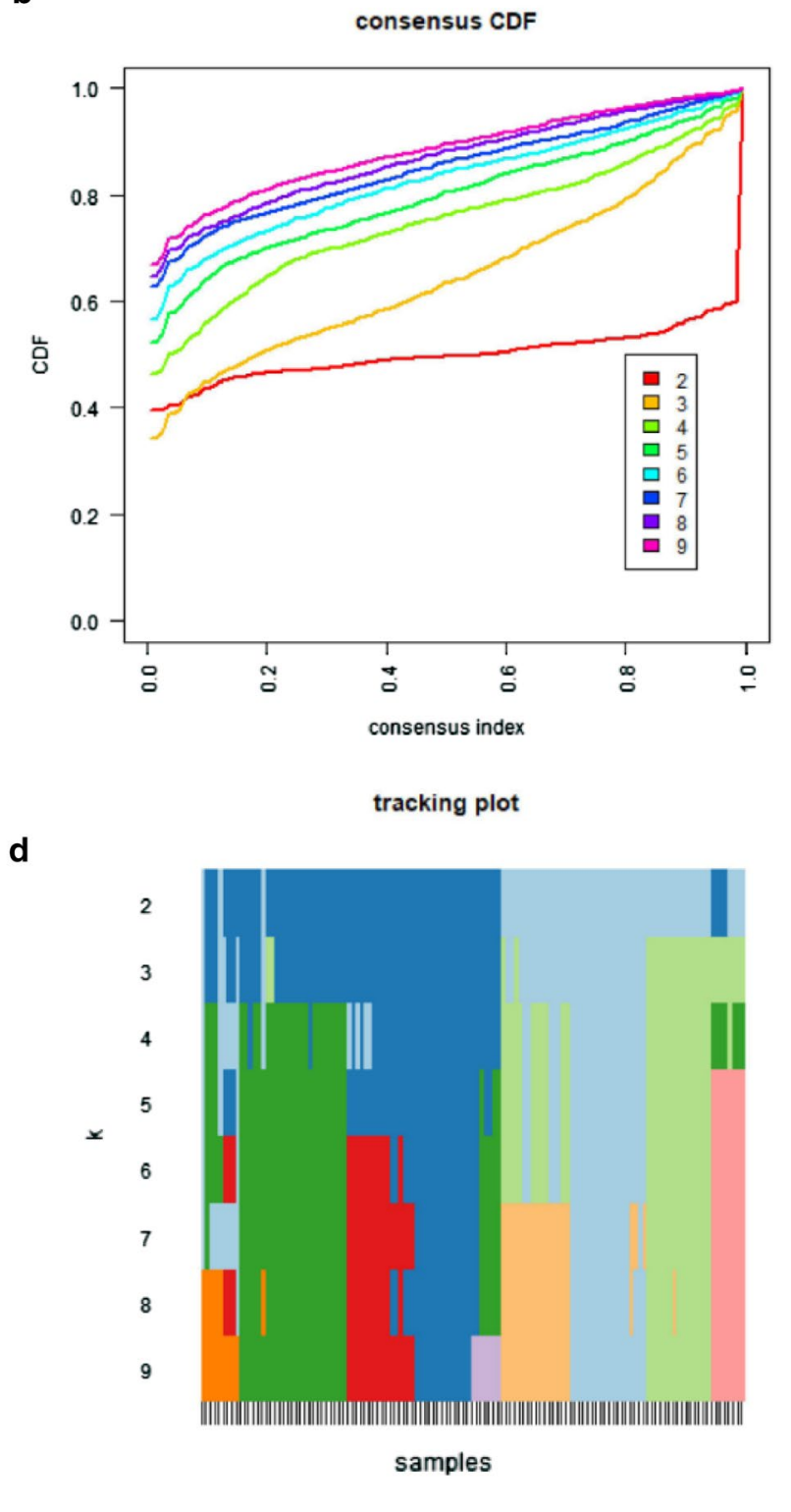

Fig. 6 Identification of consensus clusters by immune cells in GSE50081. a Consensus clustering matrix for $k=2$; $\mathbf{b}$ consensus clustering cumulative distribution function (CDF) for $\mathrm{k}=2-9$; $\mathbf{c}$ relative change in area under CDF curve for $\mathrm{k}=2-9$; $\mathbf{d}$ the tracking plot for $\mathrm{k}=2-9$

Our study showed there were 4 immune cells played vital roles in early-stage LUAD. Among the 4 identified prognostic immune cells in our study, Th2 cells and NK CD56dim cells are protective factors, in contrast to TFH and Mast cells. Lorvik et al. [26] have reported that Th2 cells and DPT cells interact each other to facilitate the escape of urological cancer, while it reported that Th2 cells were protective factors in gastric tumor [27], these suggest that $\mathrm{TH} 2$ cells may play different roles in diverse tumors. A previous study indicated that IL-15-primed
NK cel ls could survive in the ROS-rich tumor microenvironment, which is conducive to those smokers with lung cancer [28]. A Randomized Phase II Clinical Trial showed TKD/IL2-activated-NK cells were beneficial to advanced NSCLC patients after radiochemotherapy [29]. Zhenxing Guo et al. also showed that circulating Tfh (cTfh) was of great importance for the pathogenesis of NSCLC patients [30]. It reported that Mast cells (MC) was involved in the regulation of innate and adaptive immune responses [31], and a growing number of studies 

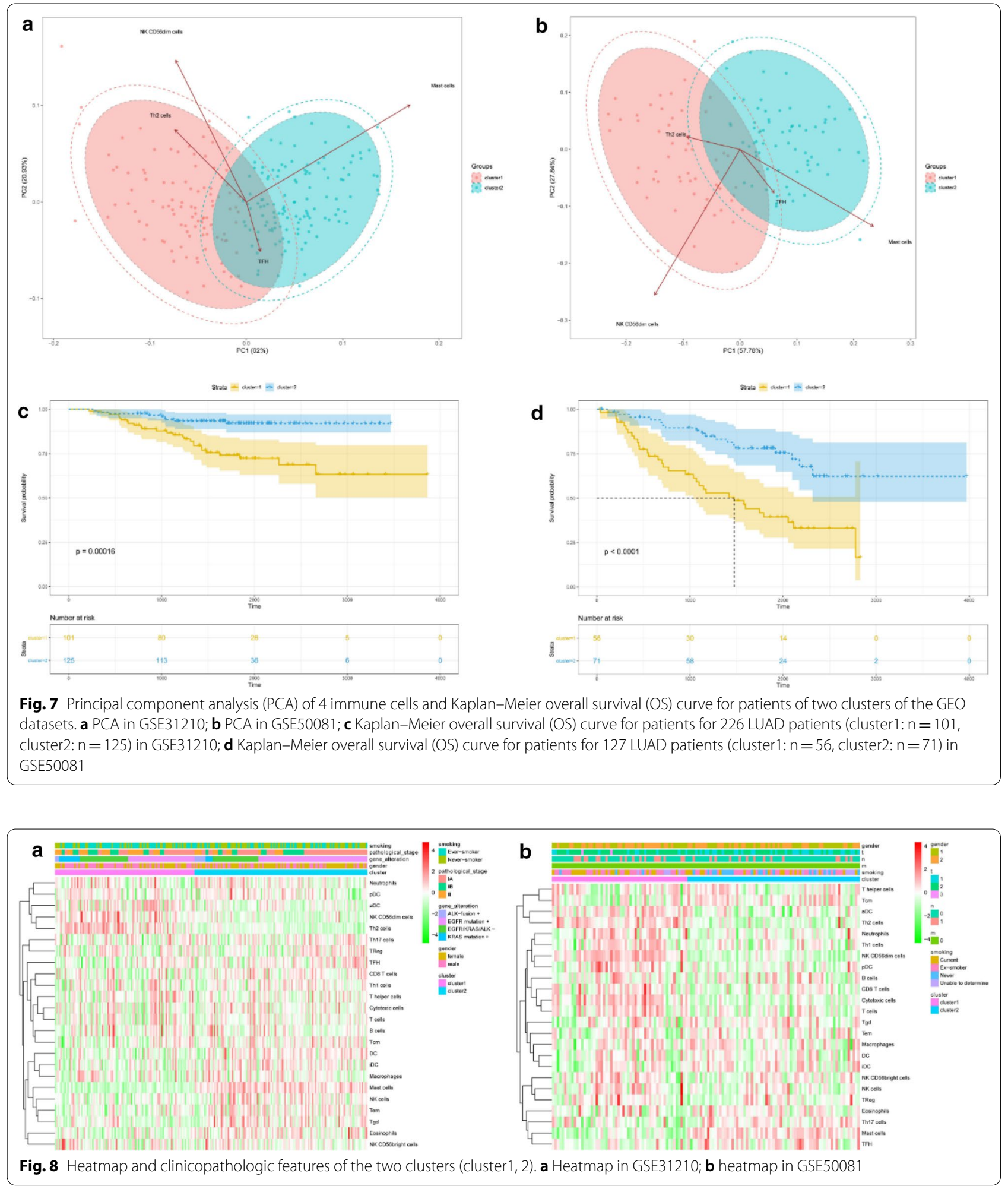

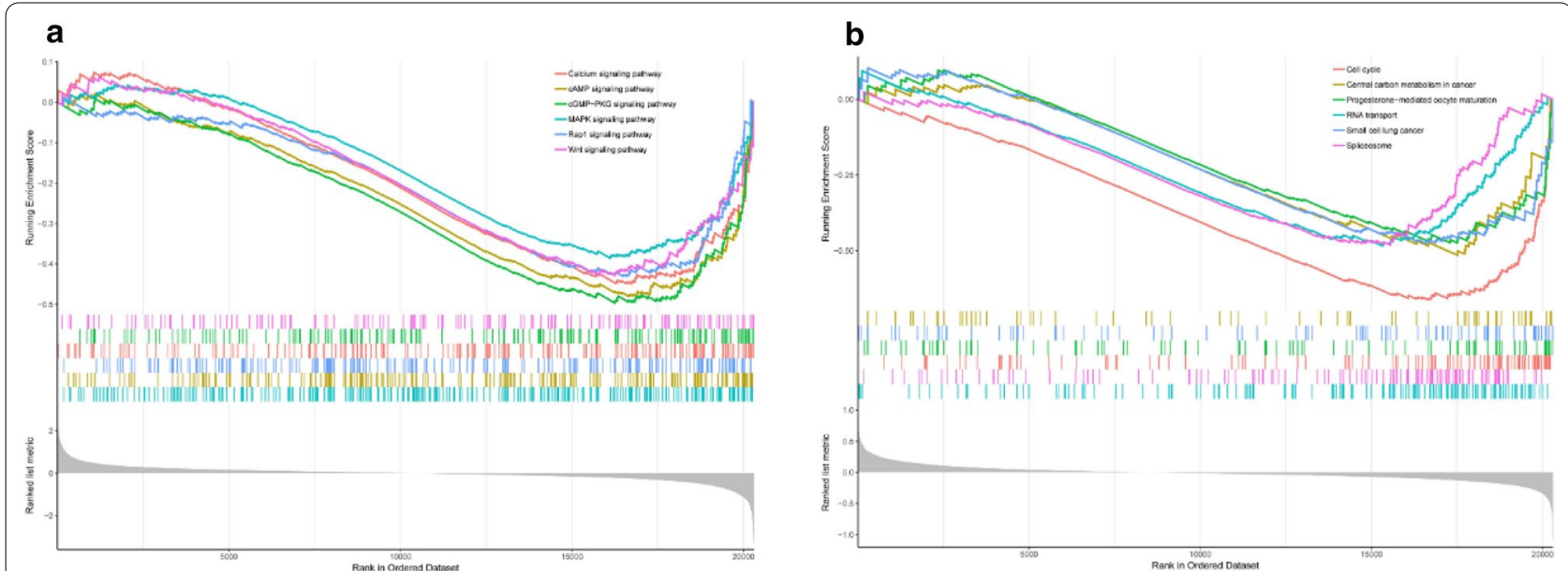

Fig. 9 Gene set enrichment analysis (GSEA). a the top 6 enriched pathways in GSE31210; b the top 6 enriched pathways in GSE50081

show that mast cells play a vital role in antineoplastic immunity in recent years $[32,33]$.

In the past, when exploring cancer-related lncRNAs, most studies only focused on the prognosis and predictive ability of a single molecule, but the process of tumor development and metastasis is often the result of a synergistic effect of multiple molecules.

Table 1 Pathways involved in both datasets (GSE31210 and GSE50081) of Gene Set Enrichment Analysis (GSEA)

\begin{tabular}{lll}
\hline No & ID & Description \\
\hline 1 & hsa05144 & Malaria \\
2 & hsa00982 & Drug metabolism_cytochrome P450 \\
3 & hsa04610 & Complement and coagulation cascades \\
4 & hsa04726 & Serotonergic synapse \\
5 & hsa04014 & Ras signaling pathway \\
6 & hsa05206 & MicroRNAs in cancer \\
7 & hsa04218 & Cellular senescence \\
8 & hsa01200 & Carbon metabolism \\
9 & hsa04114 & Oocyte meiosis \\
10 & hsa04914 & Progesterone-mediated oocyte maturation \\
11 & hsa03013 & RNA transport \\
12 & hsa03440 & Homologous recombination \\
13 & hsa03460 & Fanconi anemia pathway \\
14 & hsa01230 & Biosynthesis of amino acids \\
15 & hsa04115 & p53 signaling pathway \\
16 & hsa00051 & Fructose and mannose metabolism \\
17 & hsa00240 & Pyrimidine metabolism \\
18 & hsa03008 & Ribosome biogenesis in eukaryotes \\
19 & hsa04110 & Cell cycle \\
20 & hsa03430 & Mismatch repair \\
21 & hsa03410 & Base excision repair \\
22 & hsa03030 & DNA replication \\
\hline & &
\end{tabular}

We identified 5 hub genes (LINC00857, LINC01116, DRAIC, LINC01140 and XIST) signature performing a good prediction in early-stage LUAD patients. To date, more and more lncRNAs have been discovered and some of them are well characterized in various cancers. But the 5 lncRNAs remain uncharacterized in earlystage LUAD, while they have been reported to varying degrees in lung cancer or other diseases by previous studies. A study [34] showed that LINC00857 promoted the progression of LUAD, it regulated the cell proliferation, glycolysis and apoptosis of LUAD cells by targeting miR-1179/SPAG5 axis. Another study showed that LINC00857 regulated the progression of lung cancer through cell cycle regulation [35], there was also a study indicated that cell cycle, Hippo, TGF $\beta$, and p53 pathway alterations were associated with poor diseasefree survival (DFS) in early-stage LUAD [36], these results consistent with our results that cell cycle and p53 signaling pathway were the predicted pathways. LINC01116 was overexpressed in various tumors, such as osteosarcoma, nasopharyngeal carcinoma and some others [37-39], another study found that LINC01116 was overexpressed in LUAD tissues and cell lines and suggested that LINC01116 may act as an oncogene in LUAD [40]. And LINC01116 also contributed to gefitinib resistance of NSCLC cells [41]. Marina et al. demonstrated that LINC01140 was an important regulator of proliferation and inflammation in human lung fibroblasts [42]. LINC01140 may be a potential biomarker for the prognosis of patients with breast cancer [43] and gastric cancer [44]. However, there has no study show the connection between LINC01140 and LUAD. XIST was associated with chemotherapy resistance in non-small cell lung cancer (NSCLC) [45], and it also can expedite the progression of LUAD [46]. As for 

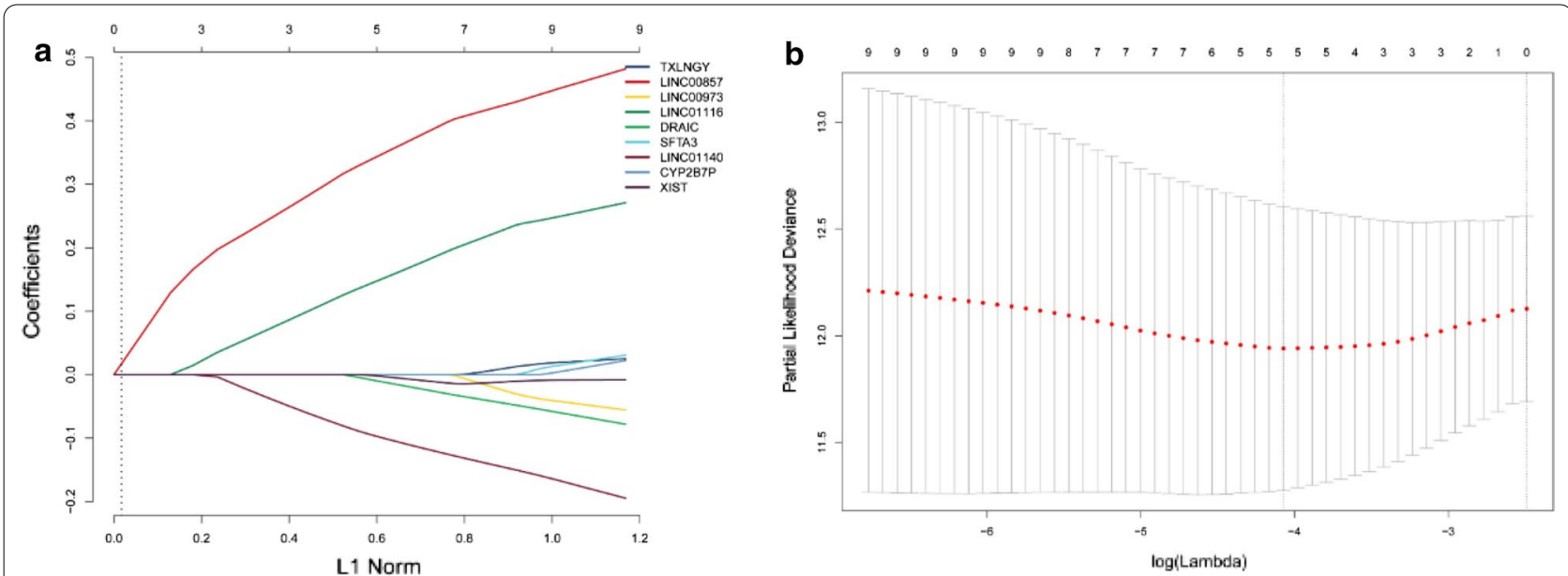

Fig. 10 a Distribution of the LASSO coefficients for 9 IncRNAs; $\mathbf{b}$ partial likelihood deviation of the LASSO coefficient distribution. Vertical dashed lines indicate lambda

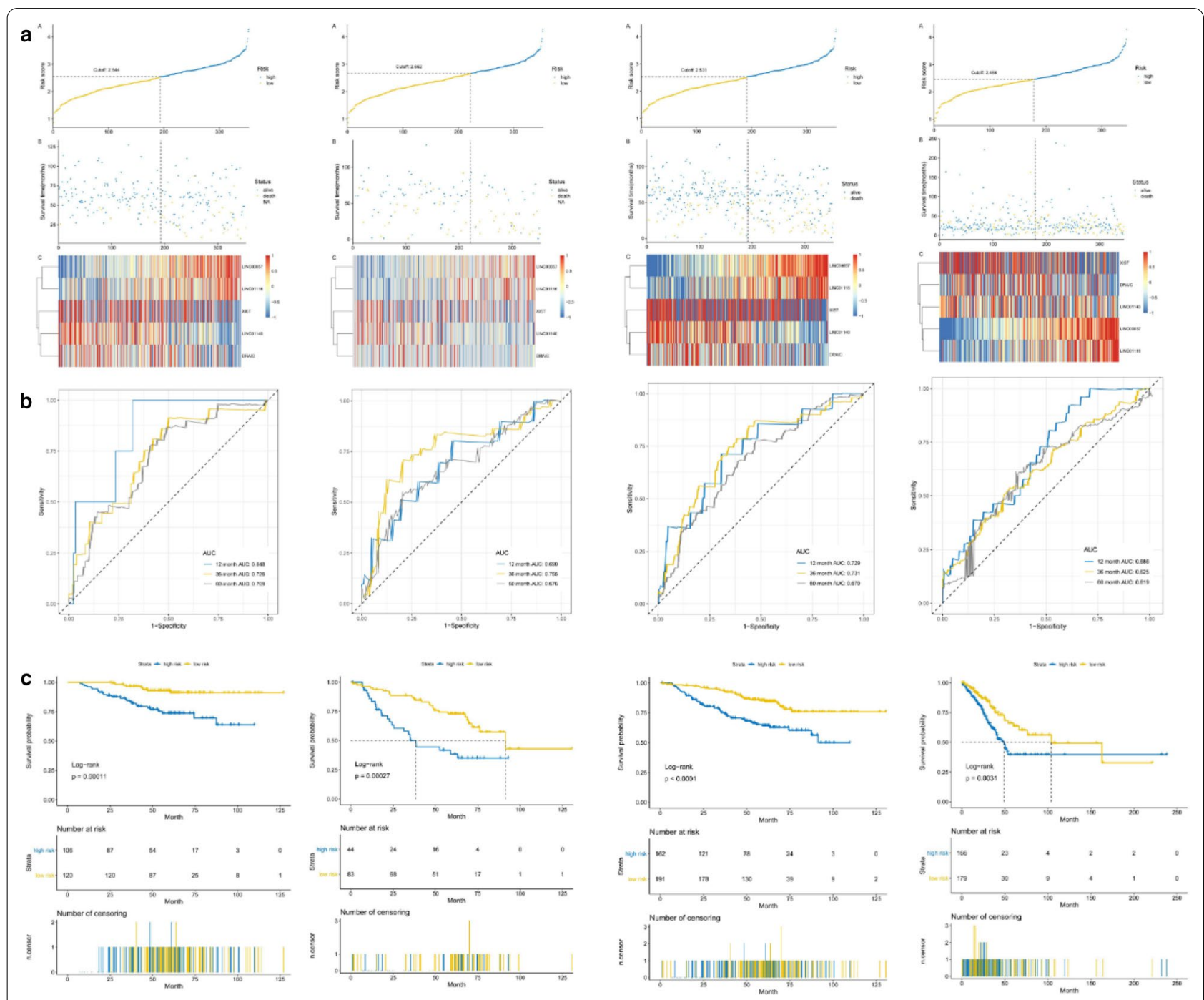

Fig. 11 Risk score, heatmap of IncRNA, time-dependent ROC analysis, and Kaplan-Meier curve of the 5-IncRNA signature in indifferent datasets. a Risk score, heatmap of IncRNA; b Time-dependent ROC analysis; $\mathbf{c}$ Kaplan-Meier curve. It was GSE31210 set, GSE50081 set, GSE31210+ GSE50081 set and TCGA set from the left to right. AUC area under the ROC curves, ROC receiver operating characteristic, TCGA The Cancer Genome Atlas 


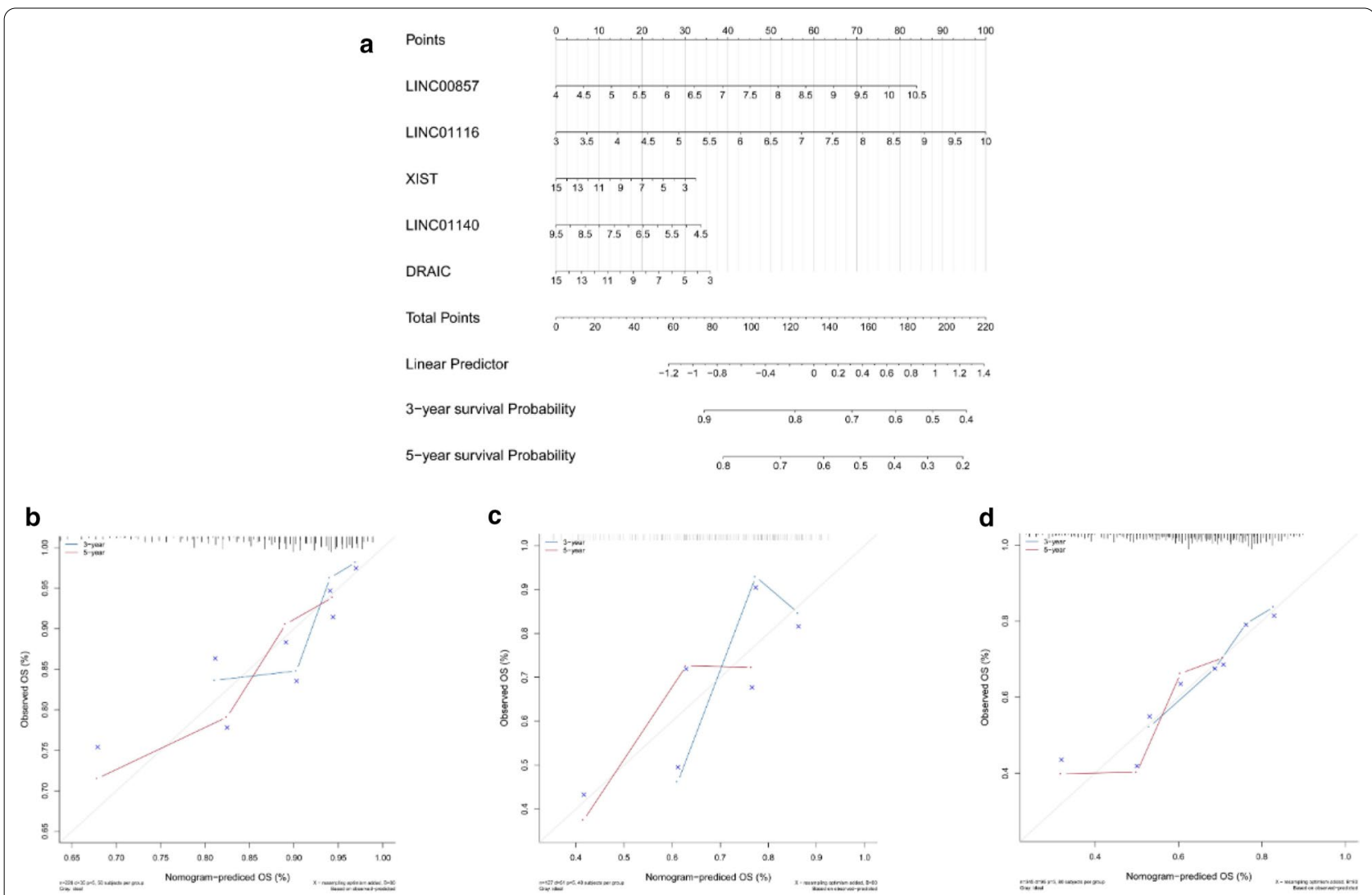

Fig. 12 a The nomogram-predicted OS for LUAD patients; b-d the calibration curves of GSE31210 dataset, GSE50081 dataset and TCGA dataset. The 3-y survival probability curve is the blue line, the 5-y survival probability curve is the red line, and the ideal curve is gray. LUAD, lung adenocarcinoma; OS, overall survival; TCGA, The Cancer Genome Atlas

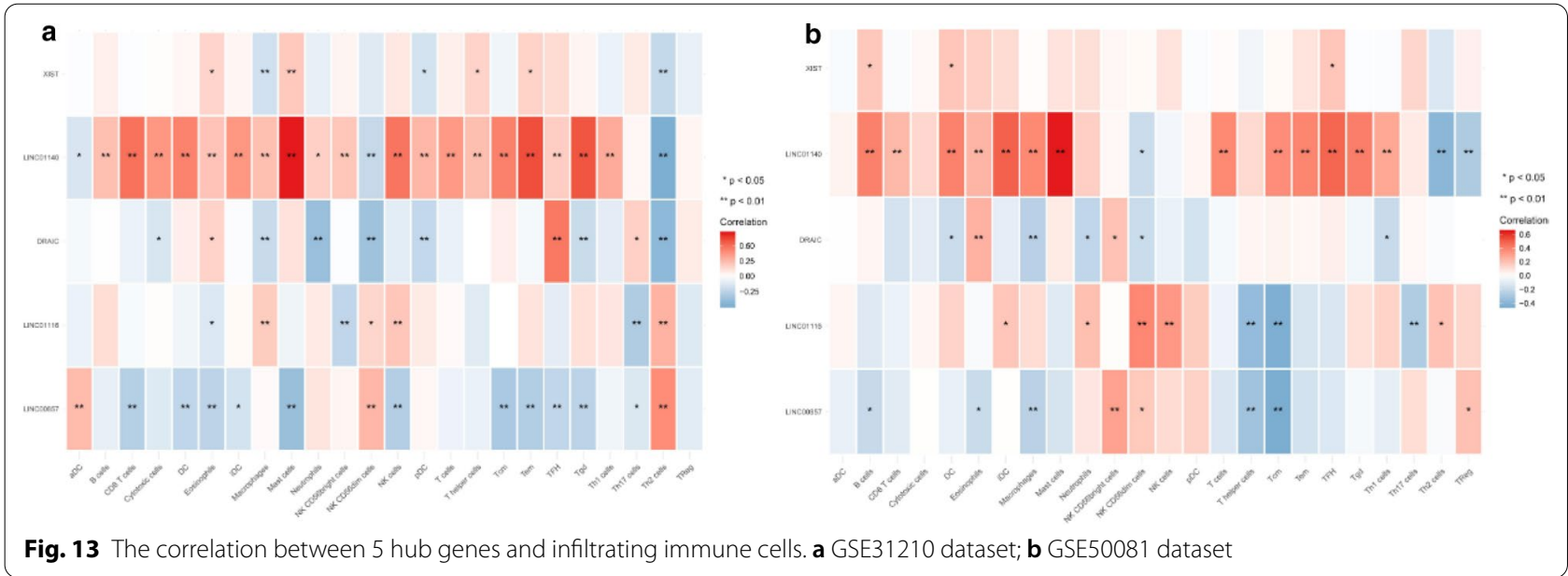

DRAIC, it was reported inhibiting prostate cancer progression through suppression of NF- $\mathrm{kB}$ activation.

The above 5 lncRNAs have been reported to varying degrees in lung cancer or other diseases. And this is the first time that we have linked lncRNAs with immune infiltration to predict the prognosis of early-stage LUAD. Through our research results, we can see that DEGs affect the prognosis of early-stage LUAD may involve many pathways, and it highlighted that lncRNA may serve as prognostic molecular markers and therapeutic 
target for patients with early-stage LUAD, and it requires further research to explore the specific mechanisms.

\section{Conclusion}

In this study, we identified 4 kinds of immune infiltrating cells related to the prognosis of early-stage LUAD, and also successfully established a novel 5 immune-related lncRNA signature for predicting patients prognosis with early-stage LUAD, these results may provide new ideas of molecular mechanism for future research and relevant target therapy of early-stage LUAD.

\section{Acknowledgements}

We thank the researchers who gave their data for this analysis. And we also would like to acknowledge the GEO and TCGA database.

\section{Authors' contributions}

(I) Conception and design: WY and KD; (II) Administrative support: WY; (III) Provision of study materials or patients: WY and KD; (IV) Collection and assembly of data: LM and KD; (V) Data analysis and interpretation: WY, KD and RT; (VI) Manuscript writing: all authors. All authors read and approved the final manuscript.

\section{Funding}

The study was supported by National Natural Science Foundation of China (Grants No. 81900053) and Project of Hunan Provincial Department of Science and Technology (Grants No. B20180778).

\section{Availability of data and materials}

The datasets supporting the conclusions of this article are available in the Gene Expression Omnibus (GEO) database and the cancer genome atlas (TCGA) database.

\section{Declarations}

Ethics approval and consent to participate

The data of this study are from GEO and TCGA database, and do not involve animal experiments and human specimens, no ethics-related issues.

\section{Consent for publication}

Not applicable.

\section{Competing interests}

The authors declare that they have no competing interests.

\section{Author details \\ ${ }^{1}$ Department of Thyroid Surgery, The Second Xiangya Hospital, Central South University, Changsha 410000, Hunan, China. ${ }^{2}$ Department of Urology, Xiangya Hospital, Central South University, Changsha 410000, Hunan, China. ${ }^{3}$ Depart- ment of Neurology, The Second Xiangya Hospital, Central South University, Changsha 410000, Hunan, China. ${ }^{4}$ Department of Respiratory Medicine, National Key Clinical Specialty, Xiangya Hospital, Central South University, Changsha 410000, Hunan, China.}

Received: 10 January 2021 Accepted: 19 March 2021

Published online: 26 March 2021

\section{References}

1. Bray F, Ferlay J, Soerjomataram I, Siegel RL, Torre LA, Jemal A. Global cancer statistics 2018: GLOBOCAN estimates of incidence and mortality worldwide for 36 cancers in 185 countries. CA Cancer J Clin. 2018;68(6):394-424
2. Torre LA, Bray F, Siegel RL, Ferlay J, Lortet-Tieulent J, Jemal A. Global cancer statistics, 2012. CA Cancer J Clin. 2015;65(2):87-108.

3. Siegel RL, Miller KD, Jemal A. Cancer statistics, 2020. CA Cancer J Clin. 2020;70(1):7-30.

4. Denisenko TV, Budkevich IN, Zhivotovsky B. Cell death-based treatment of lung adenocarcinoma. Cell Death Dis. 2018;9(2):117.

5. Kim HC, Jung CY, Cho DG, Jeon JH, Lee JE, Ahn JS, et al. Clinical characteristics and prognostic factors of lung cancer in Korea: a pilot study of data from the Korean Nationwide Lung Cancer Registry. Tuberc Respir Dis (Seoul). 2019;82(2):118-25.

6. Fridman WH, Pagès F, Sautès-Fridman C, Galon J. The immune contexture in human tumours: impact on clinical outcome. Nat Rev Cancer. 2012;12(4):298-306.

7. Peng WX, Koirala P, Mo YY. LnCRNA-mediated regulation of cell signaling in cancer. Oncogene. 2017;36(41):5661-7.

8. Xie W, Yuan S, Sun Z, Li Y. Long noncoding and circular RNAs in lung cancer: advances and perspectives. Epigenomics. 2016;8(9):1275-87.

9. Soudyab M, Iranpour M, Ghafouri-Fard S. The role of long non-coding RNAs in breast cancer. Arch Iran Med. 2016;19(7):508-17.

10. Wang J-Z, Xiang J-J, Wu L-G, Bai Y-S, Chen Z-W, Yin X-Q, et al. A genetic variant in long non-coding RNA MALAT1 associated with survival outcome among patients with advanced lung adenocarcinoma: a survival cohort analysis. BMC Cancer. 2017;17(1):167.

11. Wu D-M, Wang S, Wen X, Han X-R, Wang Y-J, Shen M, et al. LncRNA SNHG15 acts as a ceRNA to regulate YAP1-Hippo signaling pathway by sponging miR-200a-3p in papillary thyroid carcinoma. Cell Death Dis. 2018;9(10):947.

12. Chen M, Liu B, Xiao J, Yang Y, Zhang Y. A novel seven-long non-coding RNA signature predicts survival in early stage lung adenocarcinoma. Oncotarget. 2017;8(9):14876-86.

13. Lin T, Fu Y, Zhang X, Gu J, Ma X, Miao R, et al. A seven-long noncoding RNA signature predicts overall survival for patients with early stage nonsmall cell lung cancer. Aging (Albany NY). 2018;10(9):2356-66.

14. Abdel Ghafar MT, Gharib F, Abdel-Salam S, Elkhouly RA, Elshora A, Shalaby $\mathrm{KH}$, et al. Role of serum Metadherin mRNA expression in the diagnosis and prediction of survival in patients with colorectal cancer. Mol Biol Rep. 2020;47(4):2509-19.

15. Ghafar MTA, Gharib F, Al-Ashmawy GM, Mariah RA. Serum high-temperature-required protein A2: A potential biomarker for the diagnosis of breast cancer. Gene Rep. 2020;20:100706.

16. AbdelGhafar MT, Allam AA, Darwish S. Serum HOX transcript antisense RNA expression as a diagnostic marker for chronic myeloid leukemia. Egypt J Haematol. 2019;44(2):91-7.

17. El-Guindy DM, Wasfy RE, Abdel Ghafar MT, Ali DA, Elkady AM. Oct4 expression in gastric carcinoma: association with tumor proliferation, angiogenesis and survival. J Egypt Natl Canc Inst. 2019;31(1):3.

18. Habib EM, Nosiar NA, Eid MA, Taha AM, Sherief DE, Hassan AE, et al. Circulating miR-146a expression predicts early treatment response to imatinib in adult chronic myeloid leukemia. J Investig Med. 2021;69(2):333-7.

19. Colaprico A, Silva TC, Olsen C, Garofano L, Cava C, Garolini D, et al. TCGAbiolinks: an R/Bioconductor package for integrative analysis of TCGA data. Nucleic Acids Res. 2016;44(8):e71.

20. Qian C, Li H, Chang D, Wei B, Wang Y. Identification of functional IncRNAs in atrial fibrillation by integrative analysis of the IncRNA-mRNA network based on competing endogenous RNAs hypothesis. J Cell Physiol. 2019;234(7):11620-30.

21. Du Z, Fei T, Verhaak RGW, Su Z, Zhang Y, Brown M, et al. Integrative genomic analyses reveal clinically relevant long noncoding RNAs in human cancer. Nat Struct Mol Biol. 2013;20(7):908-13.

22. Finotello F, Trajanoski Z. Quantifying tumor-infiltrating immune cells from transcriptomics data. Cancer Immunol Immunother. 2018;67(7):1031-40.

23. Bindea G, Mlecnik B, Tosolini M, Kirilovsky A, Waldner M, Obenauf AC, et al. Spatiotemporal dynamics of intratumoral immune cells reveal the immune landscape in human cancer. Immunity. 2013;39(4):782-95.

24. Yu G, Wang L-G, Han Y, He Q-Y. clusterProfiler: an R package for comparing biological themes among gene clusters. OMICS. 2012;16(5):284-7.

25. Sauerbrei W, Royston $\mathrm{P}$, Binder $\mathrm{H}$. Selection of important variables and determination of functional form for continuous predictors in multivariable model building. Stat Med. 2007;26(30):5512-28.

26. Lorvik KB, Hammarström C, Fauskanger M, Haabeth OAW, Zangani M, Haraldsen $\mathrm{G}$, et al. Adoptive transfer of tumor-specific Th2 cells eradicates 
tumors by triggering an in situ inflammatory immune response. Cancer Res. 2016;76(23):6864-76.

27. Wang M, Li Z, Peng Y, Fang J, Fang T, Wu J, et al. Identification of immune cells and mRNA associated with prognosis of gastric cancer. BMC Cancer. 2020;20(1):206.

28. Whiteside TL. NK cells in the tumor microenvironment and thioredoxin activity. J Clin Invest. 2020;130:5115-7.

29. Multhoff G, Seier S, Stangl S, Sievert W, Shevtsov M, Werner C, et al. Targeted natural killer cell-based adoptive immunotherapy for the treatment of patients with NSCLC after radiochemotherapy: a randomized phase II clinical trial. Clin Cancer Res. 2020;26:5368-79.

30. Guo Z, Liang H, Xu Y, Liu L, Ren X, Zhang S, et al. The role of circulating $T$ follicular helper cells and regulatory cells in non-small cell lung cancer patients. Scand J Immunol. 2017;86(2):107-12.

31. Galli SJ, Nakae S, Tsai M. Mast cells in the development of adaptive immune responses. Nat Immunol. 2005;6(2):135-42.

32. Bulfone-Paus S, Bahri R. Mast cells as regulators of T Cell responses. Front Immunol. 2015;6:394

33. Oldford SA, Marshall JS. Mast cells as targets for immunotherapy of solid tumors. Mol Immunol. 2015:63(1):113-24.

34. Wang L, Cao L, Wen C, Li J, Yu G, Liu C. LncRNA LINC00857 regulates lung adenocarcinoma progression, apoptosis and glycolysis by targeting miR1179/SPAG5 axis. Hum Cell. 2020;33(1):195-204.

35. Wang L, He Y, Liu W, Bai S, Xiao L, Zhang J, et al. Non-coding RNA LINC00857 is predictive of poor patient survival and promotes tumor progression via cell cycle regulation in lung cancer. Oncotarget. 2016;7(10):11487-99.

36. Zhou J, Sanchez-Vega F, Caso R, Tan KS, Brandt WS, Jones GD, et al. Analysis of tumor genomic pathway alterations using broad-panel nextgeneration sequencing in surgically resected lung adenocarcinoma. Clin Cancer Res. 2019;25(24):7475-84.

37. Zhang ZF, Xu HH, Hu WH, Hu TY, Wang XB. LINC01116 promotes proliferation, invasion and migration of osteosarcoma cells by silencing p53 and EZH2. Eur Rev Med Pharmacol Sci. 2019;23(16):6813-23.

38. Chen Z, Tao Q, Qiao B, Zhang L. Silencing of LINC01116 suppresses the development of oral squamous cell carcinoma by up-regulating microRNA-136 to inhibit FN1. Cancer Manag Res. 2019;11:6043-59.
39. Xing H, Sun H, Du W. LINC01116 accelerates nasopharyngeal carcinoma progression based on its enhancement on MYC transcription activity. Cancer Med. 2020;9(1):269-77.

40. Zeng L, Lyu X, Yuan J, Wang W, Zhao N, Liu B, et al. Long non-coding RNA LINC01116 is overexpressed in lung adenocarcinoma and promotes tumor proliferation and metastasis. Am J Transl Res. 2020;12(8):4302-13.

41. Wang H, Lu B, Ren S, Wu F, Wang X, Yan C, et al. Long noncoding RNA LINC01116 contributes to gefitinib resistance in non-small cell lung cancer through regulating IFI44. Mol Ther Nucleic Acids. 2020;19:218-27.

42. Hadjicharalambous MR, Roux BT, Csomor E, Feghali-Bostwick CA, Murray $L A$, Clarke DL, et al. Long intergenic non-coding RNAs regulate human lung fibroblast function: Implications for idiopathic pulmonary fibrosis. Sci Rep. 2019;9(1):6020

43. Li D, Li L, Cao Y, Chen X. Downregulation of LINC01140 is associated with adverse features of breast cancer. Oncol Lett. 2020;19(2):1157-64.

44. Song P, Jiang B, Liu Z, Ding J, Liu S, Guan W. A three-IncRNA expression signature associated with the prognosis of gastric cancer patients. Cancer Med. 2017;6(6):1154-64.

45. Tian L-J, Wu Y-P, Wang D, Zhou Z-H, Xue S-B, Zhang D-Y, et al. Upregulation of long noncoding RNA (InCRNA) X-inactive specific transcript (XIST) is associated with cisplatin resistance in non-small cell lung cancer (NSCLC) by downregulating MicroRNA-144-3p. Med Sci Monit. 2019;25:8095-104.

46. Rong $\mathrm{H}$, Chen B, Wei $\mathrm{X}$, Peng J, Ma K, Duan S, et al. Long non-coding RNA XIST expedites lung adenocarcinoma progression through upregulating MDM2 expression via binding to miR-363-3p. Thorac Cancer. 2020;11(3):659-71.

\section{Publisher's Note}

Springer Nature remains neutral with regard to jurisdictional claims in published maps and institutional affiliations.
Ready to submit your research? Choose BMC and benefit from:

- fast, convenient online submission

- thorough peer review by experienced researchers in your field

- rapid publication on acceptance

- support for research data, including large and complex data types

- gold Open Access which fosters wider collaboration and increased citations

- maximum visibility for your research: over $100 \mathrm{M}$ website views per year

At BMC, research is always in progress.

Learn more biomedcentral.com/submissions 